\title{
Simulation of Matched Field Processing Localization Based on Empirical Mode Decomposition and Karhunen-Loève Expansion in Underwater Waveguide Environment
}

\author{
Qiang Wang ${ }^{1}$ and Qin Jiang ${ }^{2}$ \\ ${ }^{1}$ Department of Quality and Safety Engineering, China Jiliang University, Hangzhou, Zhejiang 310018, China \\ ${ }^{2}$ College of Metrology and Measurement Instrument, China Jiliang University, Hangzhou, Zhejiang 310018, China \\ Correspondence should be addressed to Qiang Wang, qiangwang@cjlu.edu.cn
}

Received 1 July 2009; Revised 26 November 2009; Accepted 22 February 2010

Academic Editor: Frank Ehlers

Copyright (C) 2010 Q. Wang and Q. Jiang. This is an open access article distributed under the Creative Commons Attribution License, which permits unrestricted use, distribution, and reproduction in any medium, provided the original work is properly cited.

\begin{abstract}
Mismatch problem has been one of important issues of matched field processing for underwater source detection. Experimental use of MFP has shown that robust range and depth localization is difficult to achieve. In many cases this is due to uncertainty in the environmental inputs required by acoustic propagation models. The paper presents that EMD (Empirical mode decomposition) processing underwater acoustic signals is motivated because it is well suited for removing specific unwanted signal components that may vary spectrally. And the Karhunen-Loève expansion is applied on sample covariance matrix to gain a relatively uncorrupted signal. The EMD denoising scheme is combined with Karhunen-Loève expansion to improve underwater target localization performance of matched field processing (MFP). The robustness and effectiveness of the proposed method is tested by the benchmark cases numerical simulation when there had large environmental parameter uncertainties of the acoustic waveguide.
\end{abstract}

\section{Introduction}

Matched field processing has been extensively explored for use in detecting and localizing underwater sources. MFP uses ocean propagation models to account for multipath when generating replica vectors [1-3]. Typically, matched field source localization involves fitting measured narrowband vertical array outputs with versions of the field, predicted by a full wave deterministic normal mode model, for a set of hypothesized source ranges and depths [4]. If accurate environmental information is available, sources can be localized in range, depth, and bearing. Under practical circumstances, ocean environmental parameters such as sound speed profile, water depth, and bottom density, may have significant spatial and temporal variability. But a major difficulty facing this approach is that the localization process is extremely sensitive to errors in the assumed propagation model and array calibration. Accurately modeling multipath can also yield mismatch reduction and detection gains as compared to direct-path beamformers. Because of the limitation of inaccurate measurement as well as all kinds of noises and perturbs in underwater environment, it is inevitable for us to receive the desirable signal with noise and have the uncertainties in modeling real ocean environment [2]. As a result it would be better to find a new robust matched field processor based on denoising scheme.

MFP exploits the complex multipath structure to generate the signal replica or the so-called steering vector. The steering vector is the spatial point source response of the medium (Green's function), thus depending on not only the source location but also the environmental parameters. It is very common that the assumed environmental parameters differ from the true ones, and thus sensitivity to mismatch is the most important liability with matched-field methods [1].

To overcome the mismatch problem, many researchers have proposed some robust algorithms, and sector-focused processing was applied to the test data cases devised for the MFP. Sector-focused processor located the source with robustness similar to the replica correlator processor, but with the higher resolution characteristic of Minimum 
variance distortionless response (MVDR) [3]. A broadband MAP estimator for robust MFP termed the wideband optimum uncertain field processor (OUFP) was presented in [4]. A contrast-maximized optimization scheme was introduced to the MFP in order to reduce the sidelobe level of ambiguity surfaces [5]. The Karhunen-Loève expansion, also called feature extraction method, has been proposed with an improvement of robustness on environmental mismatch. It is one of robust MFP algorithm, which is based on the eigenvector estimation [6]. The MFP results were presented in real ocean experimental data using KarhunenLoève expansion and MVDR with white noise constrain [7]. The coherent white noise constraint processor has also been shown through simulation to perform better than the conventional and MVDR estimators at low SNR [8].

The last two decades have seen tremendous activity in the development of new mathematical and computational tools based on multiscale ideas (wavelet). Then Ding and Gong had also proposed multiresolution processing method for source detection and localization. It improves the MFP ambiguity surface performance with analysis of shallow water trial data [9]. Based on wavelet transform and feature extraction scheme, a robust MFP was proposed by G. Gong and X. Gong [10]. The main drawback of this method is that a mother wavelet has to be defined a priori. But in the end, it would seem that the wavelet family is specifically related to the analyzed dataset because certain authors have systematically chosen different wavelet families for underwater signal analyses.

Recently, a new data-driven technique, referred to as empirical mode decomposition (EMD), has been introduced by Huang et al. [11] for analyzing data from nonstationary and nonlinear processes. The EMD has received more attention in terms of applications and interpretations [12].

The major advantage of the EMD is that the basis functions are derived from the signal itself. Hence, the analysis is adaptive in contrast to the traditional methods where the basis functions are fixed. The EMD is based on the sequential extraction of energy associated with various intrinsic time scales of the signal, starting from finer temporal scales (high-frequency modes) to coarser ones (low-frequency modes). The total sum of the intrinsic mode functions (IMFs) matches the signal very well and, therefore, ensures completeness [11]. To the nonlinear and nonstationary signals analysis, the EMD method gave promising results and has advantages when compared with the wavelet transform [12].

Flandrin et al. have proposed the signal-filtering method based on EMD to process the fractional Gaussian noise [12]. Boudraa and Cexus proposed the consecutive mean square error (CMSE) criteria to differentiate IMFs of main signal component and IMFs of noise, that does not require any knowledge of $y(t)$, and use the main IMFs to reconstruct signal and performed denoising functions [13].

To represent underwater target echo involving broadband noise, our main work in this paper is the application of EMD scheme to deal with underwater acoustic signals. Then based on Karhunen-Loève expansion, robust matched field processor was constructed. The robustness and effectiveness of the suggested algorithm has been illustrated through the numerical simulation of MFP benchmark shallow water data [14].

\section{Underwater Acoustic Signals Denoising Scheme Based on EMD}

2.1. The Empirical Mode Decomposition. Traditional data analysis methods, like Fourier and wavelet-based method, require some predefined basis functions to represent a signal. The EMD relies on a fully data-driven mechanism that does not require any a priori known basis. It is especially well suited for nonlinear and nonstationary signals, such as underwater acoustic signals.

The EMD consists of the decomposition of the original signal in successive modes. The decomposition does not require specific vectors: the signal is decomposed on itself. Obtaining a mode from the original signal is called the sifting process. The decomposition of the signal $s$ is written as

$$
s(n)=\sum_{i=1}^{M} c_{i}(n)+T_{M}(n)
$$

with $c_{i}$ being the $i$ th mode of the signal. $c$ is also called intrinsic mode functions (IMF), and $T_{M}$ is the residue.

The result of the EMD produces $M$ IMFs and a residue signal. The lower-order IMFs capture fast oscillation modes while higher-order IMFs typically represent slow oscillation modes. If we interpret the EMD as a time-scale analysis method, lower-order IMFs and higher-order IMFs correspond to the fine and coarse scales, respectively.

The decomposition following the sifting process is complete; that is, the sum of all IMFs is exactly equal to the original signal. These IMFs are deduced from the signal's extrema. Each IMF has to verify the following two conditions: (1) the number of maxima and minima has to be the same or having a difference \pm 1 , and (2) the envelope from the minima and the envelope from the maxima must have a null mean. In the end, the residue has no more than two extrema. Because the number of the signal extrema is finite, it is important to note that the number of IMF is consequently finite, regardless of the features of the signal to be analyzed.

Selecting certain IMF is equivalent to filtering the signal. And the filtered signal is the result of the sum of the selected IMF [12]. The highband and lowband filtered signal will be, respectively, defined as

$$
s_{h f, k}(n)=\sum_{i=1}^{k} c_{i}(n), \quad s_{b f, k}(n)=\sum_{i=k}^{M+1} c_{i}(n),
$$

if we note $c_{M+1}[n]=T_{M}[n]$.

2.2. EMD Denoising Scheme. Noise is always present in recorded underwater acoustic signals and is contingent upon meteorological conditions, underwater noise from human activities, signal propagation, echoes, and electronic characteristics of the material for recording. The EMD does not use any predetermined filter or wavelet function and it is a fully data-driven method. 
The EMD involves the decomposition of a given signal into a series of IMFs, through the sifting process, each with distinct time scale. The EMD method can decompose any complicated signal into its IMFs which reflect the intrinsic and reality information of the signal. The performance of the denoising methods is evaluated from the CMSE: EMD has the equivalent filter bank structure with binary wavelet [12]. The signal is decomposed as (1). Each IMF stands for certain frequency band signal information, such as (2) , and small scale IMF stands for high-frequency component, means spiking signal and noise. Large-scale IMF stands for low-frequency band signal.

The EMD method is employed for decomposing the input signal into IMFs $\left(\mathrm{IMF}_{1}, \ldots, \mathrm{IMF}_{N}\right.$, where $N$ is the number of IMFs). These IMFs are soft-thresholded, yielding $t \mathrm{IMF}_{1}, \ldots, t \mathrm{IMF}_{N}$, which are thresholded versions of the original components. The filtered signal is obtained as a linear summation of thresholded IMFs.

A smooth version of the input data can be obtained by thresholding the IMFs before signal reconstruction: EMDsoft [13]. Donoho and Johnstone have proposed a universal threshold for removing added Gaussian noise given by $\tau_{j}$ [15]:

$$
\tau_{j}=\sigma_{j} \sqrt{2 \log (L)}, \quad \sigma_{j}=\frac{\mathrm{MAD}_{j}}{0.6745},
$$

where $\sigma_{j}$ is the noise level of the $j$ th IMF, and $L$ stands for the length of IMF. The $\mathrm{MAD}_{j}$ represents the absolute median deviation of the $j$ th IMF and is defined by

$$
\operatorname{MAD}_{j}=\operatorname{Median}\left\{\left|\operatorname{IMF}_{j}(t)-\operatorname{Median}\left\{\operatorname{IMF}_{j}(t)\right\}\right|\right\} .
$$

Instead of using a global thresholding, level-dependent thresholding uses a set of thresholds, one for each IMF (scale level). The soft-thresholding method shrinks the IMF samples towards zero as follows:

$$
c_{j}(t)= \begin{cases}\operatorname{IMF}_{j}(t)-\tau_{j} & \text { if }\left|\operatorname{IMF}_{j}(t)\right| \geq \tau_{j}, \\ 0 & \text { if }\left|\operatorname{IMF}_{j}(t)\right|<\tau_{j} .\end{cases}
$$

2.3. MFP Based on K-L Expansion after Denoising Scheme. Karhunen-Loève expansion method was applied to estimate eigenvector by Seong and Byun to build robust MFP in [6]. They applied empirical orthogonal function in matched field processing area and used it to estimate the eigenvectors that constitute the field in an ocean acoustic waveguide. For received field data $P$, snapshot averaged sampling covariance matrix, $\mathbf{R}$, can be decomposed as follows:

$$
\mathbf{R}=\sum_{i=1}^{\text {snapshot }} \mathbf{P} \mathbf{P}^{H}=\sum_{i=1}^{N} \lambda \mathbf{r}_{i} \mathbf{r}_{i}{ }^{H}
$$

We used eigenvector decomposition of snapshotaveraged sampling covariance matrix in order to construct the signal vector $\mathbf{P}$, and then received signal vector which is averaged over snapshots is represented as [7]

$$
\mathbf{P}=\sum_{i=1}^{N} \sqrt{\lambda_{i}} \mathbf{r}_{i}
$$

In (7), where $\lambda$ is the simulated snapshot $\mathbf{r}_{i}(1 \leq i \leq N)$ number, $\mathbf{r}_{i}$ are the eigenvectors of the covariance matrix of received signal, and superscript $H$ denotes complex conjugate transpose.

Seong and Byun have proved that the eigenvector with relatively large eigenvalues can be said to be the main features of the pressure field generated by a source located at the assumed replica position. Then eigenvalue decomposition was applied to $\mathbf{R}$ and gains the eigenvector corresponding to the largest eigenvalue.

As mentioned in $[2,16]$, this eigenvector can be used to represent the desirably received signal. For the real data situation where multisnapshots are given, if the EMD denoising for each snapshot $\mathbf{r}_{i}(1 \leq i \leq N)$ can be performed before $\mathbf{R}$ is determined, a better data processing ability may be obtained.

Conveniently, the eigenvector $\mathbf{r}$ corresponding to maximum eigenvalue represents the desirably received signal. To achieve the radiated signal from the sources EMD is performed with respect to $\mathbf{r}$ and the reduced noise $\hat{\mathbf{r}}$ of desirably received signal is defined as

$$
\widehat{\mathbf{r}}=\mathbf{r}-\mathbf{r}^{\prime},
$$

where $\mathbf{r}^{\prime}$ is the reconstructed signal of $\mathbf{r}$ obtained from EMD denoising scheme. It is known that most replicas obtained from possible candidate environment positions contain model errors because of inaccurate environment parameters, therefore replica vector $\mathbf{p}$ also is considered to consist of noise and uncorrupted signal, and the reduced noise $\hat{\mathbf{p}}$ of replica signal is defined as

$$
\hat{\mathbf{p}}=\mathbf{p}-\mathbf{p}^{\prime},
$$

where $\mathbf{p}^{\prime}$ is the reconstructed signal of $\mathbf{p}$ after EMD denoising.

The new MFP processor is constructed by EMD denoising scheme and Karhunen-Loève expansion, which is presented as

$$
\text { EMD-MFP }=\left(\mathbf{r}^{\prime H} \mathbf{p}^{\prime}\right)^{N / 2} \cdot\left(\hat{\mathbf{r}}^{H} \hat{\mathbf{p}}\right) \text {. }
$$

where - denotes inner product. This processor can be illuminated that if the signal factors $\mathbf{r}^{\prime}$ and $\mathbf{p}^{\prime}$ are perfectly matched as well as noise factors $\hat{\mathbf{r}}$ and $\hat{\mathbf{p}}$ are perfectly matched, $\mathbf{r}$ and $\mathbf{p}$ are perfectly matched so that the source location that produces the best match between the measured and predicted fields corresponds to the true source position. In addition, the exponent $N / 2$ is used to control sidelobe level. Therefore the EMD-MFP estimates of underwater object in range $r$ and depth $z$ are obtained by solving the optimization problem:

$$
\{\tilde{r}, \tilde{z}\}=\underset{\{(r, z) \in D\}}{\arg \max }\left(\mathbf{r}^{H H} \mathbf{p}^{\prime}\right)^{N / 2} \cdot\left(\hat{\mathbf{r}}^{H} \hat{\mathbf{p}}\right),
$$

where $D$ denotes the sources of all the candidate positions.

\section{Numerical Simulation}

To validate EMD-MFP robustness and effectiveness furthermore, simulation results are discussed under conditions of 
MFP benchmark cases in [17], which supply a quantitative numerical simulation comparison of MFP performance with various schemes. The normal-mode propagation model KRAKEN was used in this study [16]. The selected cases of different kinds of mismatch situations are called briefly COLNOISE, SSPMIS, and GENLMIS. In benchmark cases, underwater source frequency is $250 \mathrm{~Hz}$ in shallow water environment, whose signals are received on a vertical array of 20 receivers spanning the water column. The first case adds colored noise, the second case introduces uncertain ocean sound speed profile (SSP) data plus white noise, and the third has both colored noise and uncertainty in virtually every environmental parameters. For the colored noise case (COLNOISE) we use a noise model that is most appropriate for treating surface noise due to breaking waves. The noise sources are then modeled as a uniform distribution of monopoles located at a small distance below the surface. The noise field is then calculated using a normal mode model to propagate the monopole sources to the receiving array. The detailed discussion is provided in [17].

The precise environment is described in [17] and the actual parameters are intended to be realistic. Figure 1(a) is the true environment parameters of ocean waveguide. Figure 1(b) is the environmental information provided for SSP mismatch case (SSPMIS) and true environment for SSPMIS $\left(c(0)=1499.4 \mathrm{~m} / \mathrm{s} c\left(D^{-}\right)=1481.6 \mathrm{~m} / \mathrm{s}\right)$. Figure $1(\mathrm{c})$ is the Ocean waveguide for general mismatch case (GENLMIS). True environment is $c(0)=1499.9 \mathrm{~m} / \mathrm{s}$, $c\left(D^{-}\right)=1478.7 \mathrm{~m} / \mathrm{s} c\left(D^{+}\right)=1574 \mathrm{~m} / \mathrm{s}, c(200)=1694 \mathrm{~m} / \mathrm{s}$, $\alpha=0.19 \mathrm{~dB} / \lambda, \rho=1.79$.

3.1. COLNOISE Case. The Bartlett processor (linear MFP) is typically the cross-correlation between data and model predictions for that data resulting in a scalar output indicating the agreement between data and model. Its ambiguity surfaces typically are incoherently averaged across frequency when performing matched field processing on a broadband source. It assumes that the true source location at each frequency remains fixed, while the sidelobes will appear at different locations at different frequencies and thus will be suppressed by the average.

Replicas of the field were computed for $50 \mathrm{~m}$ increments in range from 100 to $10000 \mathrm{~m}$, and for $2 \mathrm{~m}$ increments in depth from 1 to $100 \mathrm{~m}$. These replicas were matched with the simulated covariance data using the different beamformers. For the COLNOISE case the localization performance is very good for $40 \mathrm{~dB}$ SNR case and therefore results are only presented for the lower two SNR values.

Then, we compared the underwater target localization performance of this processor with Bartlett processor to get the source location in the low SNR case. From Figures 2(a) and 2(b), the EMD-MFP and Bartlett processor localization results are all able to accurately localize target too, when SNR is $10 \mathrm{~dB}$. The true source location range is $r=9100 \mathrm{~m}$, and depth is $z=66 \mathrm{~m}$. It is clear that EMD-MFP owns robust sidelobe suppression ability. For very low $-5 \mathrm{~dB}$ SNR case, the location results of Bartlett and EMD-MFP are in Figures $3(\mathrm{a})$ and 3(b), and the true location range equals $9700 \mathrm{~m}$, and depth is $z=58 \mathrm{~m}$. The Bartlett processor cannot locate

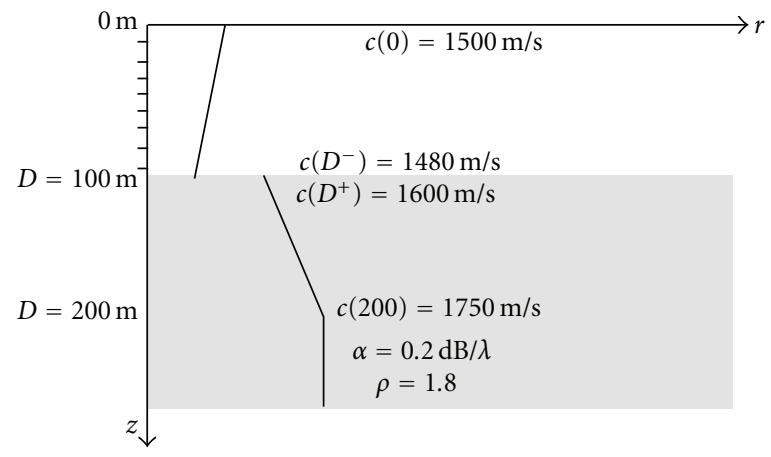

(a)

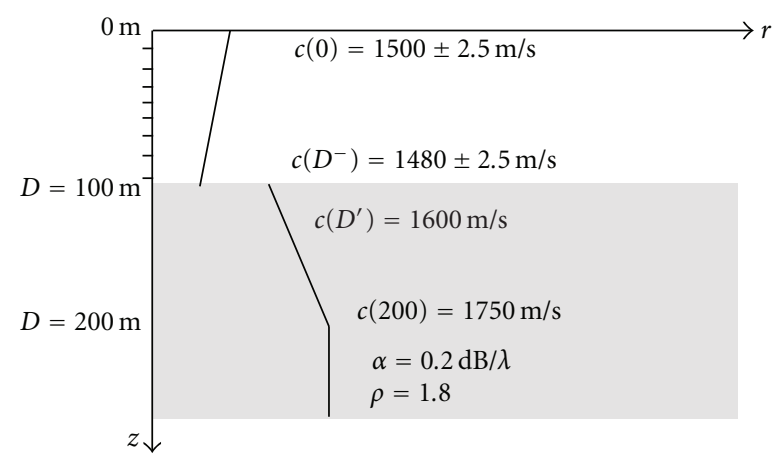

(b)

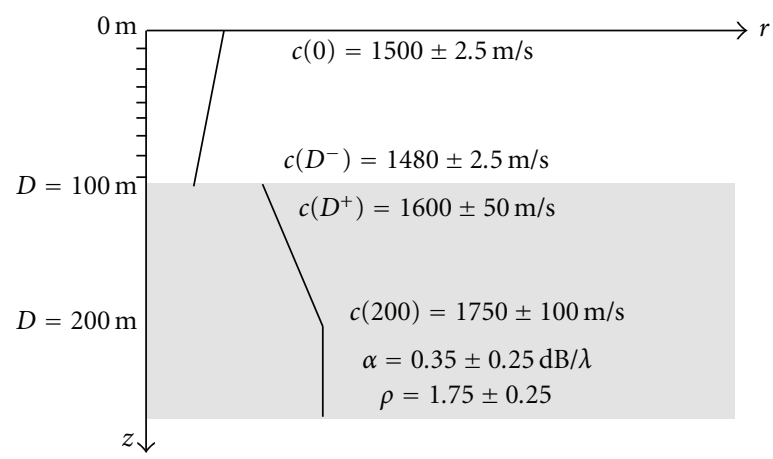

(c)

FIGURE 1: Environmental information provided for true environmental parameters (a), SSPMIS (b), and GENLMIS (c).

the source. Its sidelobes even own the same level with main lobe. Though the EMD-MFP located target correctly, it also has higher sidelobe than the localization results under SNR which is $10 \mathrm{~dB}$ condition. Naturally, the sidelobe levels tend to increase as the SNR decreases.

3.2. SSIPMIS Case. The SSPMIS represents a low level of environmental mismatch. In addition, white noise was added to the data vectors. As seen in Figure 1(a), the only source of mismatch is in the sound speed within the water column where both its gradient and mean level were randomized. Figure 4 shows the results of EMD-MFP for SSIPMIS. The 10 random samples over the environmental parameter space were used, where they are assumed as uniformly distributed over the possible interval. Under SSIPMIS (true source position $r=9300 \mathrm{~m}, z=78 \mathrm{~m}$ ) condition, for $-5 \mathrm{~dB}$ low 


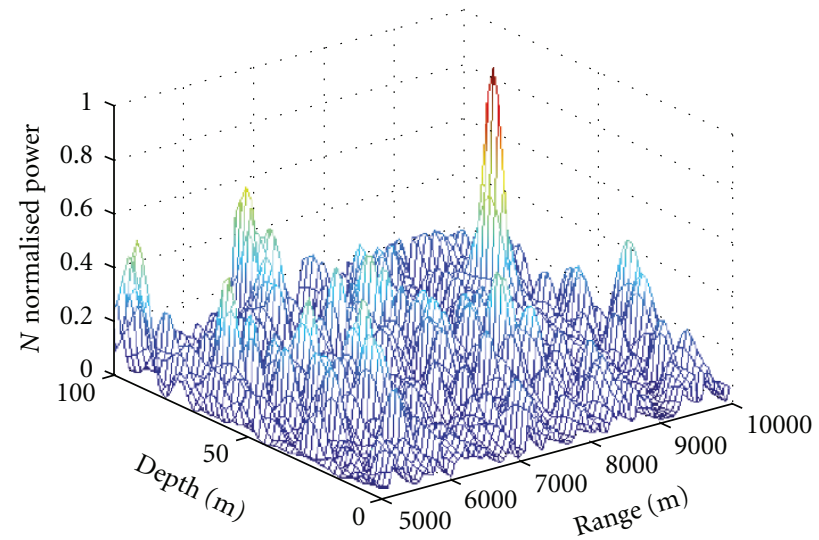

(a)

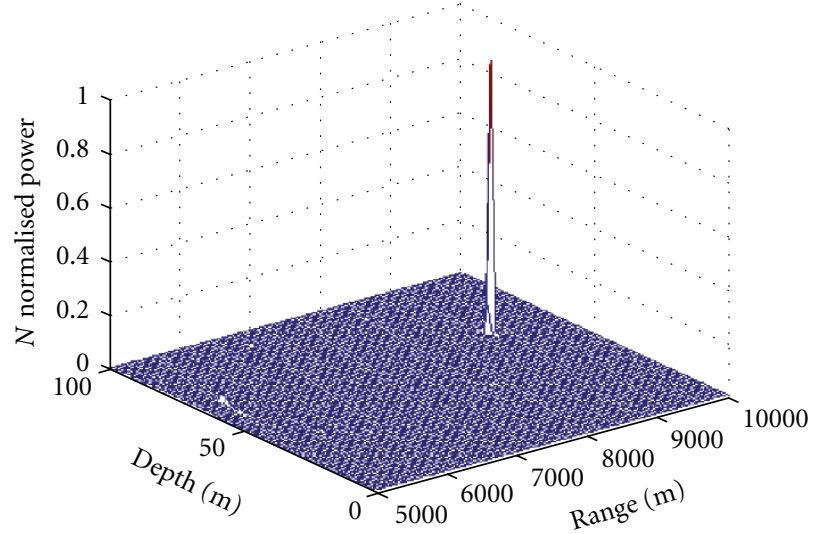

(b)

FIGURE 2: Bartlett ambiguity surface for COLNOISE with SNR of (a) and EMD-MFP ambiguity surface (b) for COLNOISE (SNR $=10 \mathrm{~dB}$ ).

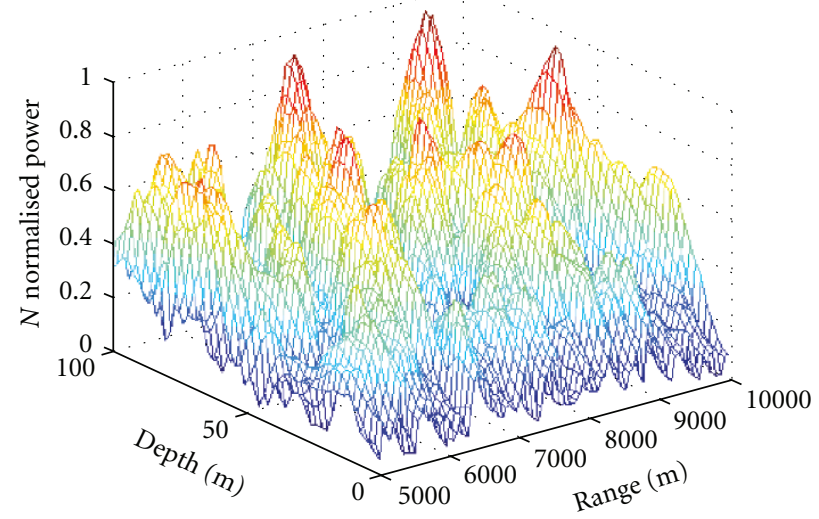

(a)

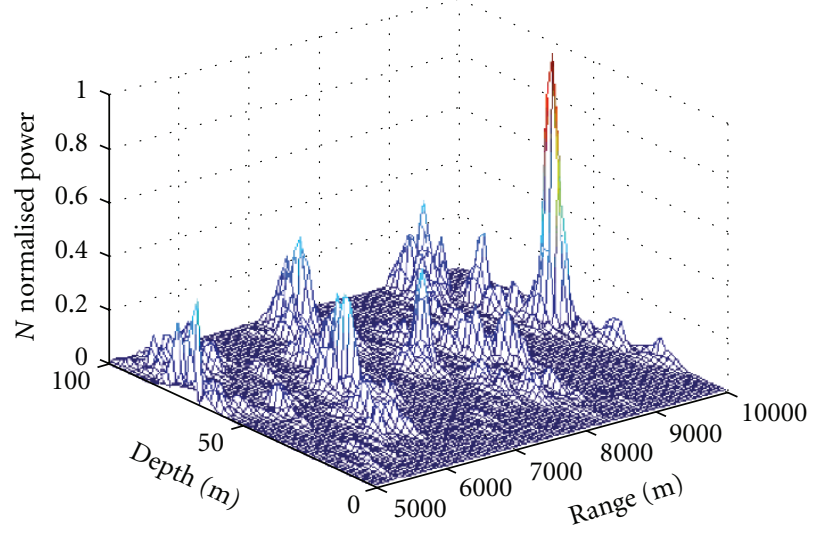

(b)

FIgure 3: Bartlett ambiguity surface (a) and EMD-MFP ambiguity surface (b) for COLNOISE ( -5 dB SNR case).

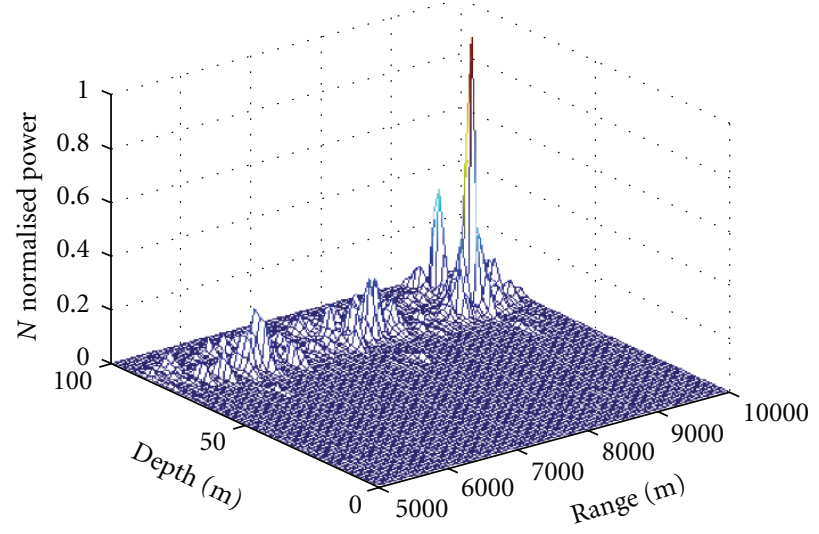

(a)

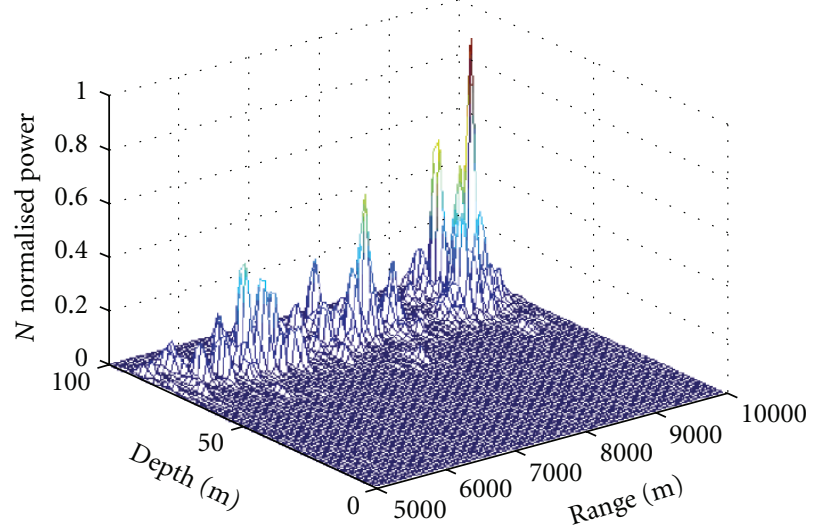

(b)

FIgURE 4: The EMD-MFP ambiguity surface (a) $10 \mathrm{~dB}$ SNR case and (b) $-5 \mathrm{~dB}$ SNR case for SSIPMIS. 


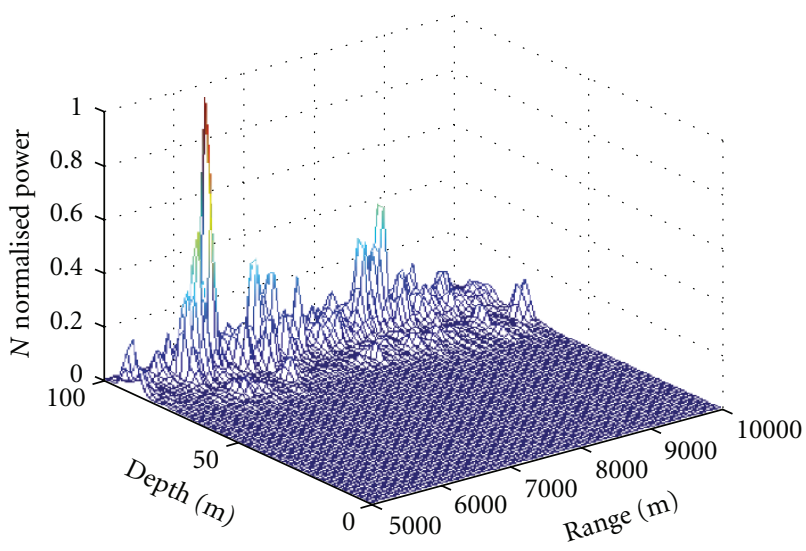

(a)

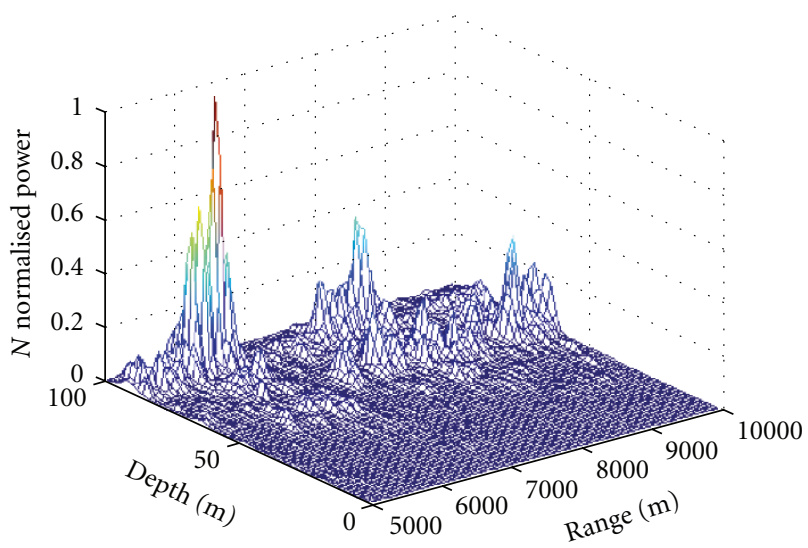

(b)

FIgUre 5: Ambiguity surface for GENLMIS using EMD-MFP localization computationally test case: $(\mathrm{a})(\mathrm{SNR}=10 \mathrm{~dB})$ and (b) $(\mathrm{SNR}=-5 \mathrm{~dB})$.

SNR case, location results are in Figure 4(a), $r=9300 \mathrm{~m}$, and $z=78 \mathrm{~m}$. Though the location is correct, the sidelobe level is high. When $\mathrm{SNR}=10 \mathrm{~dB}$ (true position $r=9300 \mathrm{~m}, z=$ $78 \mathrm{~m}$ ), the simulation results are presented in Figure 4(b). Simulation localization results are correct, but there also exist some high sidelobes in ambiguity surface.

3.3. GENLMIS Case. The general mismatch (GENLMIS) case was intended to include many problems typical of real world scenarios. It combines both mismatch and colored noise. In particular, both ocean and sediment sound speeds were randomized, the sediment attenuation and density were randomized, and the depth of the ocean/sediment interface was randomized. For GENLMIS conditions, simulation results indicate that EMD-MFP accurately localizes the source, but the sidelobe level increases with decreasing the same compared with the COLNOISE case. When SNR is $-5 \mathrm{~dB}$, we compute the target location results of the case. In Figure 5, true target source position is $r=6200 \mathrm{~m}, z=92 \mathrm{~m}$, the EMD-MFP localization results $r=6200 \mathrm{~m}$, and depth $z=92 \mathrm{~m}$ are correct. For $-5 \mathrm{~dB}$ low SNR case, EMD-MFP is also able to localize true source.
However, we saw that strong differences were evident in ambiguity surface. Noticeable difference between different SNRs is shown in Figure 5. It reveals that EMD-MFP locates the true source position under the very low $-5 \mathrm{~dB}$ SNR condition. The eigenvalues of sampling covariance matrix spread out and so signal $\mathbf{P}$ has relatively large noise components as well as source signal components. After the proposed scheme processing, the total performance of processor is satisfied under GENLMIS condition.

\section{Conclusion}

The eigenvector of sample covariance matrix corresponding to the maximum eigenvalue is seen as the desirably received signal; however this desirably received signal is still considered to contain the signal from the radiated source and noise, so EMD denoising method is performed to denoise the underwater acoustic signals.

Simulation which is similar to the environmental parameters presented in the MFP benchmark workshop was carried out, and the results showed that the proposed method owns robustness source detection performance and effective sidelobe suppression in spite of severe environmental mismatches. These results are encouraging since they imply that detailed environmental knowledge may not be a prerequisite for source localization with MFP. In GENLMIS mismatch environment, after EMD denoising, we selected the largest dominant eigenvector generated by perturbing environment to replace replica field vector in MFP that can provide robust localization performance. For relatively low-level signal, source detection was performed but peak-to-noise field ratio was low. Especially, in the test cases of slight environmental mismatches, it localized the true source location with low sidelobes.

\section{Acknowledgments}

The authors thank Dr. Gong G. Y. for her help and the anonymous reviewers for their useful comments, which lead to a better presentation of this paper. The work was supported by the National Nature Science Foundation of China under Grant 60902095 and funded by Zhejiang Provincial Natural Science Foundation of China under Grant no. Y1090672.

\section{References}

[1] A. Tolstoy, "Matched field processing: a powerful tool for the study of oceans and scatterers," in Acoustic Interactions With Submerged Elastic Structures, G. Ardéshir, M. Gérard, E. Juri, and W. Michael, Eds., vol. 5, pp. 84-111, World Science, Singapore, 2001.

[2] A. B. Baggeroer, W. A. Kuperman, and P. N. Mikhalevsky, "Overview of matched field methods in ocean acoustics," IEEE Journal of Oceanic Engineering, vol. 18, no. 4, pp. 401-424, 1993.

[3] G. B. Smith, H. A. Chandler, C. Feuillade, and D. J. Morris, "Sector focusing for robust high resolution analysis of the 
matched field processing workshop data set," Journal of Computational Acoustics, vol. 2, no. 3, pp. 199-215, 1994.

[4] J. A. Shorey and L. W. Nolte, "Wideband optimal a posteriori probability source localization in an uncertain shallow ocean environment," Journal of the Acoustical Society of America, vol. 103, no. 1, pp. 355-361, 1998.

[5] K. C. Shin and J. S. Kim, "Matched field processing with contrast maximization," Journal of the Acoustical Society of America, vol. 118, no. 3, pp. 1526-1533, 2005.

[6] W. Seong and S.-H. Byun, "Robust matched field-processing algorithm based on feature extraction," IEEE Journal of Oceanic Engineering, vol. 27, no. 3, pp. 642-652, 2002.

[7] K. S. Kim, W. Seong, and C. Lee, "Matched field processing: analysis of feature extraction method with ocean experimental data," in Proceedings of the International Symposium on Underwater Technology (UT '04), pp. 181-185, Taipei, Taiwan, 2004.

[8] C. Debever and W. A. Kuperman, "Robust matched-field processing using a coherent broadband white noise constraint processor," Journal of the Acoustical Society of America, vol. 122, no. 4, pp. 1979-1986, 2007.

[9] F. Ding and X. Y. Gong, "Multiresolution processing for source detection and localization," Acta Acustica, vol. 28, no. 1, pp. 40-44, 2003.

[10] G. Gong and X. Gong, "Robust matched field processor based on wavelet denoising and feature extraction," Chinese Journal of Electronics, vol. 16, no. 4, pp. 683-687, 2007.

[11] N. E. Huang, Z. Shen, S. R. Long, et al., "The empirical mode decomposition and the Hilbert spectrum for non-linear and non-stationary time series analysis," Proceedings of the Royal Society of London A, vol. 454, no. 1971, pp. 903-995, 1998.

[12] P. Flandrin, G. Rilling, and P. Goncalves, "Empirical mode decomposition as a filter bank," IEEE Signal Processing Letters, vol. 11, no. 2, pp. 112-114, 2004.

[13] A.-O. Boudraa and J.-C. Cexus, "EMD-based signal filtering," IEEE Transactions on Instrumentation and Measurement, vol. 56, no. 6, pp. 2196-2202, 2007.

[14] F. B. Jensen, W. A. Kuperman, M. B. Porter, and H. Schmidt, Computational Ocean Acoustics, AIP Press, Woodbury, NY, USA, 1994.

[15] D. L. Donoho and I. M. Johnstone, "Ideal spatial adaptation by wavelet shrinkage," Biometrika, vol. 81, no. 3, pp. 425-455, 1994.

[16] M. B. Porter, “The KRAKEN normal mode program," Tech. Rep. SM-245, SACLANT Undersea Research Centre, La Spezia, Italy, 1991.

[17] M. B. Porter and A. Tolstoy, "The matched field processing benchmark problems," Journal of Computational Acoustics, vol. 2, no. 3, pp. 161-185, 1994. 